\title{
Recuperación de los Destinos Turísticos Después de un Desastre: Una Evaluación del Scorecard de Resiliencia Turística para Destinos (SRTD)
}

\author{
Estefania Mercedes Basurto Cedeño* \\ Universidad Laica Eloy Alfaro de Manabí (Ecuador) \\ Lori Pennington - Gray** \\ University of Florida (USA) \\ Xavier Alberto Basurto Cedeño**** \\ Universidad Laica Eloy Alfaro de Manabí (Ecuador)
}

\begin{abstract}
Resumen: En el año 2015 se llevó a cabo un estudio de resiliencia en la ciudad de Manta usando como herramienta el Scorecard de Resiliencia Turística para Destino (SRTD). Los resultados de dicho estudio exhibieron debilidades significativas en la capacidad de respuesta del Departamento Turístico del cantón frente a escenarios de crisis que pudiesen afectar el destino. De igual manera se detectaron falencias en el portafolio de estrategias de recuperación y adaptación para escenarios probables de crisis. Ante las problemáticas encontradas los investigadores presentaron propuestas de mejoras, que no pudieron ser implementadas por el Gobierno Autónomo Descentralizado (GAD) de Manta debido a falta de presupuesto para medidas de prevención de riesgos. En abril de 2016, exactamente un año después del estudio, un terremoto de 7.8 grados en la escala de Richter sacudió la ciudad de Manta evidenciando las falencias encontradas por el SRTD. El presente trabajo realiza una valoración cuantitativa y cualitativa de la efectividad del SRTD al momento de evaluar la resiliencia de los destinos turísticos, mediante un estudio comparativo entre los resultados obtenidos en el 2015 y las problemáticas enfrentadas por el GAD de Manta después del terremoto. La finalidad de este estudio es verificar la precisión, confiabilidad y validez del SRTD al momento de evaluar la capacidad de resiliencia de los destinos turísticos, y por tanto comprobar en base a datos estadísticos que tan útil es la herramienta al momento de señalar las debilidades y fortalezas de los destinos frente a escenarios de riesgo.
\end{abstract}

Palabras Clave: Resiliencia; Scorecard; Turismo; Destinos; Terremoto.

Tourism Destination Recovery after Disaster: An Evaluation of the Tourism Disaster Resilience Scorecard for Destinations (TDRSD)

Abstract: In 2015 a resilience study was conducted in the city of Manta using the Tourism Disaster Resilience Scorecard for Destinations (TDRSD). The results showed significant weaknesses in the capacity of the Tourism Department of the destination to deal with crisis scenarios that may affect the city. The study also revealed an ambiguous portfolio of recovery and adaption strategies. The researchers presented a proposal of an improvement plan to strengthen the capacity of responseand recovery of the destination which was not implemented due to lack of budget. On April 16th, a year after the study, a 7.8 (Richter scale) earthquake struck the city of Manta, evidencing the flaws found in the study. The present study focused on the quantitative and qualitative evaluation of the TDRSD validity and efficacy when measuring the resilience scores of a tourism destination, using a mixed methodology to compare the results obtained in 2015 and the problems faced in Manta after the earthquake. The main aim of this study is to verify the precision, validity, and reliability of the TDRSD when evaluating the resilience capacity of tourism destinations, thus asserting the utility and veracity of the tool when evaluating the weakness and strengths of the destination facing risk scenarios.

Keywords: Resilience; Scorecard; Tourism; Destinations; Earthquake.

* Universidad Laica Eloy Alfaro de Manabí (Ecuador); E-mail: estefibc16@gmail.com

** University of Florida (USA); E-mail: ebasurto@ufl.edu

*** Universidad Laica Eloy Alfaro de Manabí (Ecuador); E-mail: xavier_jedy@hotmail.com 


\section{Introducción}

Muchos destinos en países en vía de desarrollo han recurrido al turismo como una herramienta que favorece el crecimiento económico y el bienestar de las poblaciones (Briedenhann y Wickens, 2004) por tal razón millones de dólares se han invertido en infraestructura turística en las últimas décadas a fin de aumentar y satisfacer la demanda turística en América Latina (Eugenio-Martin et al., 2004). Dicha inversión ha sido dirigida con la premisa de contribuir con la erradicación de la pobreza (Scheyvens, 2007) a través del trabajo generado por la actividad turística. La importancia de la actividad turística en el desarrollo económico y social de los pueblos está ampliamente documentada (McKercher, 1993; Aramberri, \& Butler, 2005; Pennington-Gray \& Pizam, 2011; Pennington-Gray et al., 2011; Biggs, 2011; Pennington-Gray et al., 2013; Basurto-Cedeno \& Pennington-Gray, 2016), y sin embargo sigue existiendo una escasa planificación en función de la disminución de riesgos del destino.

En vista de la necesidad de asegurar la sostenibilidad del sector turístico algunos destinos han optado por la adopción de marcos y modelos de gestión de riesgos (Ritchie, 2004), algunos enmarcados en el paradigma de resiliencia, el cual ha surgido como un concepto que permite aumentar la capacidad de recuperación de los destinos a la par que identifica y controla los posibles riesgos (Walker et al. 2004; Lam et al., 2012; Walker and Salt, 2012; Lew, 2014;). El marco de resiliencia proporciona un enfoque más dinámico para la planificación y el desarrollo de los destinos porque reconoce la complejidad de los sistemas turísticos (Lew, 2014). Es así que desde las organizaciones de control de turismo internacional (como la Organización Mundial de Turismo y la Secretaria de Riesgo de las Naciones Unidas) se ha recomendado que el sector turístico invierta en formas de mejorar su adaptabilidad y capacidad en respuesta a las perturbaciones (Strickland-Munro et al., 2011).

Según la Resilience Alliance, el primer paso para mejorar la resiliencia en un destino turístico encierra una investigación exhaustiva de las características del contexto ( Ruiz Ballesteros, 2011; Strickland-Munro et al., 2011; Biggs et al., 2012; Becken, 2013; Lew, 2014: Basurto-Cedeño et al., 2018) la cual debería incluir un diagnostico pormenorizado de las posibles amenazas o riesgos inherentes del sistema, así como la capacidad de preparación y respuesta frente a distintos escenarios de crisis. Es por tanto de suma importancia la adopción de una herramienta estandarizada y fiable que permita una evaluación general de la resiliencia del destino turístico y por tanto establecer un punto de referencia para desarrollar estrategias de adaptación para el mismo ( Ludin y Arbon, 2017).

Basurto y Pennington-Gray, (2016) desarrollaron el Scorecard de Resiliencia Turística para Destino (SRTD) con el objeto de contar con un instrumento que permita determinar los niveles de resiliencia de los destinos y los posibles escenarios de crisis, así como la generación de una lista exhaustiva de elementos y procesos para una rápida recuperación (Basurto y Pennington-Gray, 2016). El TDRSD se basó en diez elementos esenciales propuestos por la Oficina de las Naciones Unidas para la Reducción del Riesgo de Desastres, y se desarrolló en dos etapas: 1) un estudio de Delphi realizado con expertos en el campo de la resiliencia, la reducción del riesgo y el turismo, con el fin de ajustar los 10 esenciales de la resiliencia para que puedan encajar en un contexto turístico; y 2) la validación del instrumento a través de un estudio realizado en Manta, Ecuador (Basurto-Cedeno y Pennington-Gray, 2016).

Los resultados del estudio en Manta revelaron debilidades en los procesos de preparación y respuesta de la ciudad ante posibles escenarios de crisis, así como infraestructura turística inestable y un departamento turístico con baja capacidad de respuesta en caso de eventos catastróficos (disponibilidad recursos económicos y asesoramiento profesional). Los niveles generales de resiliencia turística para Manta fueron bajos con tal solo 108 puntos alcanzados de un total de 230 (46,96\% de resiliencia), el estudio proporcionó recomendaciones al Departamento de Turismo de Manta, para que se pudieran elaborar y aplicar ordenanzas para mejorar la puntuación general de resiliencia del destino. Desafortunadamente, las recomendaciones sugeridas no se pudieron adoptar debido a presupuesto insuficiente disponible para el período (véase Basurto-Cedeno y Pennington-Gray, 2016.)

El 16 de abril de 2016 (un año después del estudio) un terremoto de 7.8 grados en la escala Richter sacudió Manta destruyendo el centro comercial de la ciudad y afectando seriamente al sector turístico del destino. Doce hoteles colapsaron durante el terremoto, y 14 fueron demolidos después, otras estructuras turísticas (museos, aeropuertos, centros recreativos, etc.) también fueron afectadas debiendo cerrar por semanas. Las líneas de cruceros suspendieron su operación en la ciudad durante los primeros tres meses después del evento, y el destino desde entonces ha experimentado con disminución significativa de visitantes. 


\section{Resiliencia}

La resiliencia es la capacidad de un sistema para absorber perturbaciones y reorganizarse mientras cambia para volver rápidamente a su estado "normal”, es decir regresar a la misma función, estructura, e identidad previa a la perturbación (Walker, 2003). El concepto de resiliencia fue introducido en 1973 por Hollings como una nueva forma de entender los sistemas ecológicos y sus ciclos adaptativos (Walker, 2004; Folke, 2006; Hollings, 1973; Biggs, 2015; Galloping, 2006; Ruiz Ballesteros, 2011). Hollings también presentó el "concepto de resiliencia ecológica", y lo definió como el tiempo necesario para que un ecosistema regresara al equilibrio después de haber sufrido una perturbación. El concepto de resiliencia comenzó en el campo de la ciencia ecológica; sin embargo, se amplió para incluir un componente social a finales de los años noventa cuando los investigadores reconocieron que el elemento humano ha desempeñado un papel transcendental en la dinámica de un ecosistema (Folke, 206). Desde entonces, el concepto de resiliencia ha sido adoptado por varias disciplinas cada una de las cuales ha modificado su definición de acuerdo con las necesidades propias de su campo.

\subsection{Resiliencia comunitaria}

Según Cutter et al. (2008) una comunidad es la totalidad de interacciones de un sistema social que se encuentran en un espacio geográfico definido, como un vecindario, un distrito censal, una ciudad o un condado. Por lo tanto, la resiliencia comunitaria es un atributo de la población que se caracteriza por la participación activa de los miembros de la comunidad en la anticipación y respuesta a las crisis (sociales o ambientales) para recuperar toda su funcionalidad después de un evento (Norris et al., 2008). Consecuentemente, las medidas o estrategias adoptadas por la comunidad podrían asegurar la rápida recuperación de un destino, haciendo énfasis en la calidad de los vínculos poblacionales existentes. Según Norris et al. (2008), hay tres tipos de relaciones o vínculos comunitarios: (1) apoyo social,(2) participación social y (3) lazos comunitarios. El apoyo social es la conexión que existe entre las personas que están relacionadas sentimentalmente o por lazos consanguíneos; en este grupo se incluyen familiares y amigos, los cuales son fundamentales para la resiliencia comunitaria, ya que estos son los primeros proveedores de asistencia después de una crisis (Aldrich \& Meyer, 2015). El segundo tipo de relación es la participación social que se centra en los lazos que existen entre las personas que comparten intereses comunes, pero que pueden ser demográficamente diversas. Por último, los lazos comunitarios, se entienden como el capital social a nivel comunitario que se centra en la participación ciudadana en grupos y actividades comunitarias (Sherrieb y Norris, 2010).

Según Herrera y Rodríguez (2016) la resiliencia comunitaria es la capacidad de los destinos de alcanzar un nuevo estado "normalidad" después de que una crisis importante haya perturbado el equilibrio de la comunidad; por lo tanto, bajo esta premisa las comunidades nunca regresan al estado anterior a la crisis, sino que cambian y se adaptan a las nuevas circunstancias. En algunos casos puede darse incluso que los miembros de la comunidad sean reacios a regresar a su hogar, y que opten por trasladarse a lugares que perciban como más seguros. Herrera y Rodríguez (2016) también identificaron que los pilares social, económico y ecológico son cruciales en la recuperación de los destinos y que por tanto deben ser monitoreadas después de un desastre para evaluar si la comunidad está mostrando evidencia de resiliencia.

A pesar de las diferencias existentes entre las diversas conceptualizaciones de resiliencia comunitaria, de forma general existe consenso en cuanto al papel clave que juegan los conceptos de vulnerabilidad y capacidad de adaptación en la recuperación post crisis y por tanto en la resiliencia de los destinos turísticos (Jopp et al., 2010). el uso de un cuadro de mando estandarizado para medir la resiliencia podría proporcionar un primer diagnóstico para identificar debilidades en el plan de gestión de crisis, y las estrategias de adaptación para la comunidad ( Ludin y Arbon, 2012) y por tanto contribuir en el estudio de la vulnerabilidad y la creación de capacidad de recuperación.

\section{Scorecard de Resiliencia Turística para Destinos (SRTD)}

Es habitual el uso de los Scorecards (cuadros de mando) en las evaluaciones de resiliencia de destino, ya que constituyen un punto de partida para hacer un diagnóstico de los destinos y así poder empezar procesos que ayuden a aumentar la capacidad de respuesta en caso de emergencia (Cutter, 2016). El SRTD es una herramienta basada en los diez elementos esenciales de resiliencia propuestos por la Oficina de las Naciones Unidas para la Reducción del Riesgo de Desastres (Basurto-Cedeno y Pennington-Gray, 2016) e implementado en la ciudad de Manta en el año 2015 con nueve esenciales: (1) organización y coordinación, (2) presupuesto, (3) planta de gestión de riesgos, (4) infraestructura 
crítica, (5) instalaciones turísticas,(6) programas de educación y formación, (7) ecosistemas naturales, (8) gestión de alertas y emergencias, y (9) comercialización después de la crisis.

Todos los esenciales del SRTD están subdivididos en elementos (Tabla 1), en total la herramienta está constituida por 46 elementos que se evalúan en base a criterios específicos y cuya ponderación varía entre 0 y 5 puntos dependiendo del nivel de planificación y los esfuerzos que el destino haya realizado para abordar dicho elemento. Además, cada criterio cuenta con una descripción precisa y una lista de características que orientan la calificación (Basurto y Pennington- Gray, 2015), de esta manera los elementos del SRTD sirven como indicadores de evaluación y priorización de las necesidades y los objetivos de una comunidad (Cutter, 2016).

Tabla 1: Elementos de la Scorecard de Resiliencia Turística para Destinos

\begin{tabular}{|c|c|}
\hline Essential Subjects & Description of item \\
\hline $\begin{array}{l}\text { (1) Organización y } \\
\text { coordinación }\end{array}$ & $\begin{array}{l}\text { 1. Acciones previas al evento } \\
\text { 2. Respuesta del evento } \\
\text { 3. Participación } \\
\text { 4. Contribuciones } \\
\text { 5. Eficacia de las asociaciones } \\
\text { 6. Eficacia de los barrios } \\
\text { 7. Inserción } \\
\text { 8. Habilidades } \\
\text { 9. Propuesta del gobierno }\end{array}$ \\
\hline (2) Presupuesto & $\begin{array}{l}\text { 1. Adecuación de la planificación financiera } \\
\text { 2. Capital } \\
\text { 3. Financiación operativa } \\
\text { 4. Disponibilidad de fondos de contingencia } \\
\text { 5. Incentivos para la organización turística } \\
\text { 6. Incentivos para la organización sin fines de lucro } \\
\text { 7. Cobertura de seguros para sitios turísticos y activos patrimoniales }\end{array}$ \\
\hline $\begin{array}{l}\text { (3) Plan de gestión de } \\
\text { riesgos }\end{array}$ & $\begin{array}{l}\text { 1. Conocimiento de los peligros } \\
\text { 2. Conocimiento de la exposición y vulnerabilidad } \\
\text { 3. Actualizaciones }\end{array}$ \\
\hline $\begin{array}{l}\text { (4) Infraestructura } \\
\text { crítica }\end{array}$ & $\begin{array}{l}\text { 1. Conocimiento del número de activos para garantizar la comunicación } \\
\text { 2. Existencia de generadores eléctricos de respaldo } \\
\text { 3. Conocimiento del número de días de pérdida de electricidad después del desastre } \\
\text { 4. Conocimiento del costo y número de días para la restauración de la electricidad después del } \\
\text { desastre } \\
\text { 5. Continuidad de la operación del Departamento de Turismo } \\
\text { 6. Continuidad de los sistemas informáticos y los datos }\end{array}$ \\
\hline $\begin{array}{l}\text { (5) Instalaciones } \\
\text { turísticas }\end{array}$ & $\begin{array}{l}\text { 1. Seguridad estructural } \\
\text { 2. Número de días de pérdida de operación }\end{array}$ \\
\hline $\begin{array}{l}\text { (6) Programas de } \\
\text { educación y } \\
\text { formación }\end{array}$ & $\begin{array}{l}\text { 1. Número de turistas capacitados } \\
\text { 2. Exposición por persona } \\
\text { 3. Eficacia de la educación } \\
\text { 4. Disponibilidad de formación } \\
\text { 5. Porcentaje de la población capacitada } \\
\text { 6. Frecuencia de entrenamiento repetido } \\
\text { 7. Formación en otros idiomas }\end{array}$ \\
\hline $\begin{array}{l}\text { (7) Ecosistemas } \\
\text { naturales }\end{array}$ & 1. Conciencia del papel del ecosistema \\
\hline $\begin{array}{l}\text { (8) Gestión de alertas y } \\
\text { emergencias }\end{array}$ & $\begin{array}{l}\text { 1. Existencia del sistema de alerta } \\
\text { 2. Eficacia en términos de porcentajes de la población alcanzada } \\
\text { 3. Existencia de un plan } \\
\text { 4. Requerimiento de equipo } \\
\text { 5. Disponibilidad de equipos } \\
\text { 6. Número de días } \\
\text { 7. Disponibilidad de cooperación } \\
\text { 8. Centro de operaciones de emergencia } \\
\text { 9. Conjunto anual de simulacros } \\
\text { 10. Eficacia de los simulacros }\end{array}$ \\
\hline $\begin{array}{l}\text { (9) Marketing post } \\
\text { crisis }\end{array}$ & 1. Planificación \\
\hline
\end{tabular}


Debido a las características del SRTD, la evaluación de cada elemento esencial se debe ejecutar a nivel gerencial (Top-Down), puesto que la información sobre la planificación estratégica y gestión de crisis, presupuestos de recuperación, etc. no es de dominio público sino que es responsabilidad de los funcionarios de la ciudad (Basurto y Pennington- Gray, 2015; Cutter, 2016). Para garantizar la validez de los resultados recabados la metodología requiere una compilación de evidencias (documentos, planos, imágenes, etc.) como requisito para asignar la puntuación para cada elemento. Por tanto, los resultados de la del SRTD permiten a los gestores de destinos identificar las debilidades y fortalezas del turismo urbano ante un desastre; constituyéndose como una herramienta de diagnóstico de la eficacia de los planes de gestión de crisis turísticas a nivel de ciudad/ municipio.

\section{El terremoto}

El 16 de abril de 2016 a las 18:58 de la hora local ecuatoriana, un terremoto de 7,8 grados en la escala de Richter y con epicentro en Pedernales (Manabí) golpeó la región litoral del Ecuador. La ola de destrucción se extendió a lo largo de la línea costera afectando principalmente a las provincias de Manabí y Esmeraldas.

Se reportaron 663 víctimas mortales, nueve personas desaparecidas, 6274 heridos, 28.775 personas en refugios y unas 7000 viviendas destruidas (Instituto Nacional de Estadísticas y Censos INEC) convirtiéndose en uno de los peores desastres que haya experimentado esta zona geográfica. Según las declaraciones de la Presidencia de la República del Ecuador, las pérdidas materiales reportadas por el terremoto alcanzaron los 3.300 millones de dólares (Instituto Nacional de Estadisticas y Cesos INEC) convirtiéndose en el sismo más destructivo y costoso de la historia ecuatoriana.

Ante las devastadoras secuelas del terremoto, muchos destinos turísticos se vieron significativamente afectados. Por ejemplo Manta reportó una cifra alta de pérdidas humanas (219) y la destrucción de gran parte de la infraestructura turística en especial en la parroquia Tarqui, la cual fue declarada como la zona más afectada o "Zona Cero".

Tarqui fue históricamente el distrito económico y hotelero para el turismo doméstico de la ciudad de Manta, pero después del terremoto la mayor parte de la infraestructura y la superestructura de la zona se perdieron. El Municipio reportó 135 negocios turísticos perdidos y 14 hoteles demolidos debido a fallas estructurales. Además, la torre de control del aeropuerto fue destruida, y varias líneas de cruceros cancelaron sus operaciones durante mayo, junio y julio de 2016. Tras el terremoto, el destino ha tratado de recuperar la infraestructura turística perdida, así como de incrementar el número de visitantes, sin embargo hasta Agosto de 2019 (ultima fecha de levantamiento de datos para el estudio) el destino sigue experimentando problemas en la zona cero. Según los funcionarios de la ciudad, el retraso en las reparaciones y renovaciones en la zona cero se debe en parte a la falta de acuerdo entre los moradores y propietarios de bienes raíces de Tarqui, por tanto, que el sector fue declarado como no adecuado para la construcción de edificaciones de más de un piso. Esta falta de consenso no es inusual para las comunidades que se recuperan de un sismo, de hecho, según Norris et al. (2008) las comunidades que experimentan eventos catastróficos pueden sufrir una pérdida de identidad en la zona afectada e incluso en toda la ciudad y región.

\section{Metodología}

El estudio adoptó un diseño mixto convergente con un componente cualitativo seguido de un cuantitativo. La primera fase del estudio incluyó la recopilación y análisis documental de publicaciones digitales y en medios de comunicación tradicionales que mencionaron el impactos del sismo del 16 de abril en la ciudad de Manta. El levantamiento de información se realizó durante el lapso de 12 meses (de abril de 2016 a abril de 2017) y tuvo como objeto precisar la gravedad del terremoto en el destino desde el punto de vista turístico. En esta fase también se llevaron a cabo entrevistas con las autoridades locales de turismo y representantes de las empresas turísticas que empezaron tres meses después del terremoto y que se prolongaron hasta agosto de 2019. La segunda fase incluyó una evaluación cuantitativa de la eficacia del SRTD al diagnosticar las debilidades y desafíos que podría enfrentar un destino turístico en caso de crisis. Siguiendo un enfoque similar al propuesto por Basurto et al., (2016) los investigadores solicitaron a los participantes que proporcionaran una puntuación de eficacia para cada elemento esencial del SRTD. 
Así la validez de la herramienta se valoró mediante un estudio comparativo de las puntuaciones obtenidas antes del terremoto y la realidad post-crisis (bajo la percepción de los participantes).

\subsection{Objetivos y Preguntas de Investigación}

El objetivo de esta investigación fue medir la puntuación de resiliencia del destino después del terremoto e identificar las coincidencias y brechas con respecto a la puntuación que se realizó un año antes del sismo, de ahí que las preguntas de investigación que impulsaron el estudio fueron:

- ¿Fue adecuada la puntuación del SRTD del 2015 con respecto a la realidad experimentada post crisis?

- ¿Cómo cambió la puntuación del SRTD después del terremoto de 2016, y que similitud o divergencia se encontró en cuanto a los resultados del 2015 ?

Con la finalidad de dar respuesta a las preguntas de investigación, se adoptó un diseño de método mixto convergente, el primer componente se llevó a cabo a través de un estudio cualitativo que incluyó un análisis de documentos y entrevistas con expertos. El análisis de documentos se realizó utilizando una recopilación de las publicaciones realizadas por los medios de comunicación y las redes sociales oficiales sobre el terremoto durante el lapso de doce meses a partir del 17 de abril de 2016. El análisis documental es una técnica que admite la recopilación, el examen y la evaluación de documentos públicos e informes anuales, lo que permite flexibilidad para incluir diferentes tipos de documentos además de material escrito (Bowen, 2009; Padgett, 2016). El estudio analizó 500 documentos escritos y audiovisuales publicados que contenían información sobre el evento (terremoto). Las fuentes incluidas en el análisis fueron (1) el sitio web y la cuenta de Twitter del Ministerio de Turismo del Ecuador, (2) la cuenta de Twitter y la cuenta de Facebook del Municipio de Manta, (3) la cuenta de Twitter de la Presidencia de la República del Ecuador, (4) El periódico El Telégrafo, de Ecuador, (5) el periódico ecuatoriano El UNIVERSO, (6) El periódico mantense El Diario, y (7) el sitio web de la BBC Chanel. Las fuentes incluidas en el análisis se encontraron pertinentes con base en la sugerencia de la Directora del Ministerio de Turismo de Ecuador Zona 4, el Decano de la Facultad de Turismo de la Universidad Laica Eloy Alfaro de Manabí, y tres expertos en gestión de riesgos y resiliencia turística. Todos los documentos examinados en el estudio fueron escritos en español.

Los criterios de búsqueda de documentos oficiales para el análisis documental estaban orientados a determinar el impacto del terremoto considerando la literatura de gestión de riesgos (Coburn et al., 1992, Boarnet, 1996, Schmidtlein et al., 2011) y por tanto se enfocaron en los siguientes aspectos: (1) las víctimas humanas, (2) el colapso de los edificios, (3) el colapso de la infraestructura turística, (4) las pérdidas económicas y (5) las pérdidas del negocio turístico. Dada la relevancia de dichos criterios también se los utilizaron para desarrollar el marco de código para el análisis de contenido.

La segunda fase del análisis cualitativo incluyó entrevistas a profundidad con cuatro miembros del Departamento de Turismo de Manta, dos profesores de turismo de la Universidad Laica Eloy Alfaro de Manabí, dos hoteleros, dos agentes de viajes, dos propietarios de restaurantes y dos miembros del Ministerio de Turismo. Las entrevistas se llevaron a cabo por separado en el lugar de trabajo de los participantes. Para este estudio, se utilizó un muestreo no probabilístico a juicio con la técnica bola de nieve. Este método de muestreo se recomienda para estudios cualitativos, donde el objetivo es recopilar información sobre las partes interesadas clave del destino.

Durante las entrevistas, se pidió a los participantes que compartieran sus experiencias durante y después del sismo tomando en consideración los nueve elementos esenciales del SRTD: (1) organización y coordinación, (2) presupuesto, (3) planta de gestión de riesgos, (4) infraestructura crítica, (5) instalaciones turísticas, (6) programas de educación y formación, (7) ecosistemas naturales, (8) gestión de alertas y emergencias, y (9) comercialización después de la crisis; así también se les solicitó que facilitaran una puntuación para cada elemento esencial.

El componente cuantitativo del estudio incluyó la comparación de los valores promedios obtenidos para el SRTD antes y después del terremoto utilizando la prueba de rango de Wilcoxon. La prueba de Wilcoxon es el equivalente no paramétrico de la prueba de t emparejada (Field, 2013) y se recomienda cuando los datos analizados no son paramétricos o en estudios con muestras pequeñas y emparejadas $(<30)$ que presentan una distribución no normal. En este estudio se utilizaron los resultados de Basurto y Pennington (2016) como las puntuaciones previas al evento, y el promedio de las valoraciones de 8 expertos en gestión de desastres y turismo en los destinos como las puntuaciones post evento. 


\section{Resultados del Estudio Cualitativo}

Para evaluar la gravedad del terremoto de Manta se llevó a cabo un análisis documental que incluyó más de 500 documentos publicados en revistas, periódicos y redes sociales. Diariamente se realizó una búsqueda utilizando las palabras claves y se seleccionaron documentos durante el período de 12 meses en base en la pertinencia del material y el tema bajo estudio. Los resultados mostraron que el número final de víctimas humanas en Manta fue de 219 personas, lo que corresponde al 33\% del total de las víctimas reportadas durante el terremoto a nivel nacional; 650 edificación fueron demolidas por el Municipio debido a su estado de precariedad post-sismo, de las cuales 26 fueron infraestructuras turísticas. Adicionalmente 135 negocios turísticos cerraron y de acuerdo con la presidencia de Ecuador y el INEC se reportaron pérdidas por más de 3000 millones de dólares entre las ciudades de Manta, Portoviejo y Pedernales. En base a la información recopilada el terremoto del 16 de abril de 2019 fue uno de los más graves que ha golpeado al país.

Paralelamente las entrevistas a expertos se centraron en comprender la percepción de los participantes con respecto a la eficacia del SRTD. La totalidad de los participantes manifestaron que la herramienta identificó de forma bastante precisa las problemáticas y fortalezas del destino ante una crisis. Uno de los participantes del Departamento de Turismo mencionó:

"Los resultados del estudio del 2015 (en el cual se empleó la SRTD) parecen ser un reflejo de lo que sucedió... definitivamente no estábamos preparados para un evento de esa magnitud... sé que teníamos una idea de cómo proceder en caso de una crisis, pero lo que sucedió estaba más allá de nuestras expectativas, nunca consideramos ese escenario".

Para otro miembro del Departamento de Turismo de Manta uno de los problemas más significativos que enfrentaron después del terremoto fue la falta de información sobre los procedimientos frente a una crisis de tal magnitud:

"Teníamos un plan de manejo de crisis para la ciudad, pero nos quedó corto... nadie esperaba ese escenario... Un terremoto de esa magnitud nunca golpeó la ciudad antes; Creo que Manta nunca contempló la posibilidad de que nos pudiera pasar de algo asi””.

Los resultados del primer estudio del SRTD realizado en Manta mostraron que la infraestructura de destino era débil en especial en la parroquia Tarqui y por tanto entre las recomendaciones se sugirió un diagnóstico de la calidad de la superestructura turística; a pesar de ello los levantamientos técnicos no se pudieron realizar por falta de presupuesto del Gobierno Autónomo Descentralizado (GAD) de la ciudad de Manta. Después del sismo se evidenció la necesidad de priorizar este tipo de diagnósticos de la infraestructura comprobando así la pertinencia del SRTD. En concordancia los entrevistados manifestaron:

\footnotetext{
"Nunca se hizo un estudio de la calidad de la infraestructura turística a pesar de que era obvio que algunos hoteles estaban muy mal construidos... en ese momento pensamos que la construcción no era estéticamente aceptable, pero no imaginamos que se fueran a caer... después del terremoto se pidió a la Universidad de las Fuerzas Armadas (ESPE) que nos ayudara a diagnosticar el estado de la infraestructura turística, especialmente del sector hotelero que seguía en pie ... sin embargo los resultados del análisis no fueron validados por falta de coordinación con el Ministerio de Desarrollo Urbano y Vivienda (MIDUVI), y se necesitó una segunda evaluación...”
}

"Perdimos el 20\% de nuestra infraestructura turística, 26 establecimientos hoteleros fueron eliminados del inventario turístico de la ciudad, 12 colapsaron durante el terremoto y 14 deben ser demolidos porque la infraestructura está comprometida".

Con respecto a la evaluación de la infraestructura hotelera la mayoría de los entrevistaron mencionaron que prefieren que se realice desde el organismo de control, ya sea el Ministerio de Turismo o el GAD de Manta:

"Yo pensé que la infraestructura de mi hotel estaba en perfecto estado, pero no fue el caso... creo que deben existir evaluaciones periódicas pero dirigidas y controladas por el GAD... yo no puedo estar contratando a un ingeniero de forma privada porque seguramente me va a decir que hay problemas para que lo contrate y gastes dinero, aunque en realidad no existan esos problemas... pienso que deben venir las universidades a solicitud del GAD o del Ministerio de Turismo y hacer un estudio imparcial y sin fines de lucro". 
En cuanto a un presupuesto de recuperación, algunos participantes manifestaron que al no contar con fondos propios o seguros el proceso de reconstrucción está avanzando lentamente por cuanto se depende de créditos de instituciones financieras, las cuales solicitan un gran número de requisitos que muchas veces no están en las manos de los miembros del sector:

"dependemos de la colaboración de las instituciones financieras para la reconstrucción del sector hotelero, y las cosas están avanzando lentamente porque la zona cero, donde la mayoría de los hoteles colapsaron, está aún bajo estudio y hay no una respuesta oficial en cuanto a la viabilidad de construcciones... sin ese estudio técnico no podemos aplicar a créditos "

Adicionalmente, las entrevistas brindaron un espacio en que los participantes pudieron expresar sus inquietudes, sugerencias y estrategias para mejorar la puntuación de resiliencia del destino. Un miembro del GAD mencionó estar trabajando con el Departamento de Bomberos para crear un plan de contingencia para pequeñas empresas con el objeto de identificar la salidas y rutas en caso de emergencias. Conjuntamente los propietarios de restaurantes mencionaron estar aumentando la conciencia de riesgo entre sus clientes utilizando señales visuales preventivas.

La mayoría de los participantes señalaron que existe la necesidad de comenzar a trabajar juntos y desarrollar estrategias de reducción de riesgos y recuperación siempre contando con el apoyo y liderazgo del Departamento de Turismo de Manta. Sin embargo ante estas iniciativas algunos miembros del GAD expresaron no estar preparados para hacer frente a este tipo de requerimientos:

"Todo el mundo nos está pidiendo capacitación, y tenemos que pedir al departamento de bomberos, o al secretario de reducción del riesgo porque no nos sentimos entrenados para hacerlo".

Finalmente, los participantes coincidieron en que la puntuación de resiliencia global alcanzada en el estudio del2015 (en el que se aplicó la SRTD) era muy precisa, por cuanto tenía grandes niveles de coincidencia con respecto a la realidad experimentada en Manta después del terremoto. De forma unánime los expertos validaron la herramienta como pertinente al momento de evaluar los niveles de resiliencia de los destinos turísticos.

\section{Resultados de la prueba de Wilcoxon}

La prueba de rango de Wilcoxon se basa en encontrar diferencias entre valores no paramétricos en base a las comparaciones de los promedios de los parámetros emparejados (Field, 2013); por esta razón se descartaron los elementos esenciales que obtuvieron las mismas puntuaciones pre y post- crisis (esenciales 8 y 10) para el análisis estadístico con el software SPSS versión 24.0.

La prueba de Wilcoxon encontró que no había una diferencia significativa a nivel estadístico ( $p=.123)$ entre las puntuaciones de los elementos esenciales tomados antes y después del evento, y por tanto se sugiere no descartar la hipótesis nula (tabla 2). La puntuación general de resiliencia previa al evento (2015) fue de 108 de 230 (46,96\%), y los resultados obtenidos en 2016 mostraron una puntuación total de 89,01 puntos equivalente al 38,7\%. Las diferencias entre las dos mediciones no fueron encontradas estadísticamente significativas por el análisis ( $\mathrm{p}=.123)$, lo que valida empíricamente la eficacia del SRTD (Tabla 1). La varianza entre las puntuaciones totales de los elementos esenciales pre y post crisis se detallan a continuación.

Tabla 2: Wilcoxon test resultados

\begin{tabular}{|lllll|}
\hline & Null Hypothesis & Test & Sig. & Decision \\
\hline 1 & $\begin{array}{l}\text { La mediana de las diferencias } \\
\text { entre el evento "después" y el } \\
\text { evento "antes" es igual a 0. }\end{array}$ & $\begin{array}{l}\text { Related-Samples } \\
\text { Wilcoxon Signed } \\
\text { Rank Test }\end{array}$ & .128 & Conservar la hipótesis nula \\
\hline
\end{tabular}

Se muestran la importancia asintótica. El nivel de significancia es .05

El esencial 1 mantiene una puntuación similar en $2016(13,64)$ a los datos iniciales tomados en el 2015 (14) con una variación mínima de .36 puntos, lo que refleja una gran coincidencia entre lo que se proyectó (pre crisis) y lo que sucedió durante el terremoto (post crisis). Por otra parte el esencial 2 reveló 
Tabla 3: Diferencias en las puntuaciones después y antes del desastre por elementos

\begin{tabular}{|c|c|c|c|}
\hline Description of item & $\begin{array}{c}\text { Puntuación antes del } \\
\text { desastre }\end{array}$ & $\begin{array}{l}\text { Puntuación } \\
\text { tras desastre }\end{array}$ & Diferencia \\
\hline 1. Acciones previas al evento & 4.00 & 3.00 & -1.00 \\
\hline 2. Respuesta del evento & 3.00 & 3.38 & 0.38 \\
\hline 3. Participación & 4.00 & 3.00 & -1.00 \\
\hline 4. Contribuciones & 0.00 & 0.50 & 0.50 \\
\hline 5. Eficacia de los gremios & 3.00 & 4.00 & 1.00 \\
\hline 6. Eficacia de los barrios & 4.00 & 4.13 & 0.13 \\
\hline 7. nserción & 0.00 & 0.00 & 0.00 \\
\hline 8. Habilidades & 1.00 & 1.75 & 0.75 \\
\hline 9. $\quad$ Propuesta del gobierno & 5.00 & 4.50 & -0.50 \\
\hline 10. Adecuación de la planificación financiera & 2.00 & 1.38 & -0.62 \\
\hline 11. Capital en el presupuesto & 0.00 & 0.25 & 0.25 \\
\hline 12. Financiación operativa & 5.00 & 4.88 & -0.12 \\
\hline 13. Disponibilidad de fondos de contingencia & 0.00 & 0.00 & 0.00 \\
\hline 14. Incentivos para la organización turística & 3.00 & 2.88 & -0.12 \\
\hline 15. Incentivos para la organización sin fines de lucro & 3.00 & 3.00 & 0.00 \\
\hline $\begin{array}{l}\text { 16. Cobertura de seguros para sitios turísticos y activos } \\
\text { patrimoniales }\end{array}$ & 1.00 & 1.25 & 0.25 \\
\hline 17. Conocimiento de los peligros & 5.00 & 3.13 & -1.87 \\
\hline 18. Conocimiento de la exposición y vulnerabilidad & 5.00 & 2.88 & -2.12 \\
\hline 19. Actualización & 5.00 & 2.25 & -2.75 \\
\hline $\begin{array}{l}\text { 20. Conocimiento del número de activos para garantizar la } \\
\text { comunicación }\end{array}$ & 5.00 & 3.63 & -1.37 \\
\hline 21. Existencia de generadores de respaldo & 3.00 & 2.63 & -0.37 \\
\hline $\begin{array}{l}\text { 22. Conocimiento del número de días de pérdida de } \\
\text { electricidad después del desastre }\end{array}$ & 3.00 & 1.88 & -1.12 \\
\hline $\begin{array}{l}\text { 23. Conocimiento del costo y número de días para la } \\
\text { restauración de la electricidad después del desastre }\end{array}$ & 0.00 & 0.63 & 0.63 \\
\hline 24. Continuidad de la operación del Departamento de Turismo & 0.00 & 0.00 & 0.00 \\
\hline 25. Continuidad de los sistemas informáticos y los datos & 5.00 & 1.75 & -3.25 \\
\hline 26. Seguridad estructural & 0.00 & 0.25 & 0.25 \\
\hline 27. Número de días de pérdida de operación & 3.00 & 1.63 & -1.37 \\
\hline 28. Número de turistas alcanzados & 1.00 & 0.88 & -0.12 \\
\hline 29. Exposición por persona & 1.00 & 0.00 & -1.00 \\
\hline 30. Eficacia de la educación & 1.00 & 0.13 & -0.87 \\
\hline 31. Disponibilidad de formación & 5.00 & 4.00 & -1.00 \\
\hline 32. Porcentaje de la población capacitada & 5.00 & 1.00 & -4.00 \\
\hline 33. Frecuencia y regularidad de entrenamientos & 4.00 & 1.00 & -3.00 \\
\hline 34. Entrenamiento en otros idiomas & 0.00 & 0.00 & 0.00 \\
\hline 35. Conciencia del rol del ecosistema & 0.00 & 0.00 & 0.00 \\
\hline 36. Existencia de un Sistema de alarma & 3.00 & 2.00 & -1.00 \\
\hline 37. Efectividad en términos de porcentaje de la población & 0.00 & 2.38 & 2.38 \\
\hline 38. Existencia de un plan & 2.00 & 2.25 & 0.25 \\
\hline 39. Necesidad de equipamiento & 0.00 & 1.50 & 1.50 \\
\hline 40. Disponibilidad de equipamiento & 2.00 & 2.63 & 0.63 \\
\hline 41. Número de días & 3.00 & 3.50 & 0.50 \\
\hline 42. Disponibilidad de cooperación & 4.00 & 3.63 & -0.37 \\
\hline 43. Centro de Operaciones para emergencias & 3.00 & 3.75 & 0.75 \\
\hline 44. Conjunto anual de simulacros & 1.00 & 0.88 & -0.12 \\
\hline 45. Eficacia de los simulacros & 1.00 & 0.75 & -0.25 \\
\hline 46. Planificar la existencia & 0.00 & 0.00 & 0.00 \\
\hline
\end{tabular}


puntuaciones más altas antes de la crisis (14 puntos en 2015) en comparación de los datos del 2016 $(13,63)$. De forma parecida elementos 4 (16 puntos en 2015 y 10,75 puntos en 2016), 5 (3 puntos en 2015 y 1,88 puntos en 2016), 7 (17 puntos en 2015 y 7 puntos) en 2016) mostraron valores inferiores en el 2016.

El esencial 3 no se tomó en cuenta para comparación estadística ya que existió una declaración oficial por parte de la alcaldía de Manta (2016) en la que se manifestaba que la ciudad no contaba con un plan de gestión de crisis. Por otra parte, con respecto al esencial 9 se evidenció una puntuación más alta $(23,25)$ en 2016 que en 2015 (19). Finalmente, los esenciales 8 y 10 mantenían la misma calificación entre ambos los períodos. El resumen de las diferencias encontradas entre las puntuaciones de cada elemento se detalla en la tabla 3.

La principal diferencia entre las puntuaciones proporcionadas por el SRTD antes y después del terremoto corresponde al esencial 3, en el cual se abordaban aspectos relacionados a la existencia y eficacia del plan de gestión de crisis.

El análisis estadístico inferencial determinó que los resultados del SRTD después del terremoto no mostraban diferencias estadísticas significativas con respecto a los resultados pre - crisis $(p=123)$. Sin embargo, la puntuación general descriptiva si mostró una variación importante con una puntuación post desastre de sólo 89,01 puntos, un 38,7\% de la puntuación máxima posible, en comparación con la primera medida tomada un año antes del terremoto (108) la cual correspondía al 46,96\%.

La interpretación de los resultados cualitativos y cuantitativos se encuentran detallados en la siguiente sección.

\section{Discusión y conclusión}

El nivel de preparación y resiliencia de un destino turístico puede ser evaluado a través de un diagnóstico inicial con el uso de herramientas especializadas como el SRTD, que permiten revelar deficiencias en los planes de manejo de riesgos.

El presente estudio ha probado empíricamente la eficacia del SRTD al momento de evaluar la preparación y resiliencia de un destino turístico. Los resultados cuantitativos fueron validados mediante la triangulación de datos cualitativos proporcionados a través de entrevistas con los actores turísticos de la ciudad de Manta y expertos en turismo y manejo de riesgo.

Se encontraron pequeñas diferencias entre las puntuaciones previas al terremoto con respecto a la realidad experimentada en el destino (post crisis) que pudieron corresponder por un lado a errores involuntarios al momento de levantar los datos o debido a limitaciones del GAD de Manta al momento de ejecutar la planificación y el presupuesto. El diagnostico del SRTD resaltó deficiencias importantes con respecto al presupuesto de preparación, calidad de la infraestructura y planificación para la recuperación post - crisis, todas la cuales se evidenciaron como las mayores dificultades que experimentó y sigue experimentando el destino.

Aunque los resultados no mostraron diferencias estadísticamente significativas, es esencial observar que hay variaciones en las puntuaciones entre los parámetros (después y antes del terremoto), por lo cual existe la necesidad de una evaluación constante de los planes para la gestión de riesgo en destinos turísticos, la misma que debe ser realizada por un ente ajeno a la gobernanza y que corrobore las puntuaciones de la autoevaluación cuando se utilice el SRTD. También se recomienda evaluaciones periódicas de control para evidenciar la evolución del destino a lo largo del tiempo, y poder contribuir con información de pertinencia en la toma de decisiones a nivel de gobernanza.

\section{Limitaciones}

El Departamento de Turismo de Manta fue el ente nominador de participantes para las entrevistas, por lo que existe la posibilidad de que las opiniones de otras partes interesadas no se hayan reflejado en estos resultados, por lo tanto, no pueden generalizarse más allá de la realidad del destino bajo estudio. Se recomienda la replicación de la herramienta en otros contextos para evaluar las limitaciones de la misma en realidades diferentes.

\section{Bibliografia}

Adger, W. N. 2006. Vulnerability. Global environmental change, 16(3), 268-281.

Adger, W. N., Hughes, T. P., Folke, C., Carpenter, S. R., \& Rockström, J. 2005. Social-ecological resilience to coastal disasters. Science, 309(5737), 1036-1039. 
Aramberri, J., \& Butler, R. (Eds.). 2005. Tourism development: issues for a vulnerable industry (Vol. 20). Channel View Publications.

Basurto-Cedeño, E. M., \& Pennington-Gray, L. 2016. Tourism disaster resilience scorecard for destinations (TDRSD): The case of Manta, Ecuador. International Journal of Tourism Cities, 2(2).

Basurto-Cedeño, E. M., Pennington-Gray, L., \& Basurto-Cedeño, X. 2018. Identificación riesgos en destinos turísticos: el primer paso para incrementar la resiliencia. TURYDES Revista Turismo y Desarrollo local sostenible, (junio).

Basurto-Cedeño, E. M., Pennington-Gray, L., \& Matthews, J. S. 2015. PRESERVACIÓN DEL PATRIMONIO CULTURAL PARA EL DESARROLLO TURÍSTICO.

Becken, S. 2013. Developing a framework for assessing resilience of tourism sub-systems to climatic factors. Annals of Tourism Research, 43, 506-528.

Berkes, F., \& Ross, H. 2013. Community resilience: toward an integrated approach. Society \& Natural Resources, 26(1), 5-20.

Berkes, F., Colding, J., \& Folke, C. (Eds.). 2008. Navigating social-ecological systems: building resilience for complexity and change. Cambridge University Press.

Biggs, D. 2011. Understanding resilience in a vulnerable industry: the case of reef tourism in Australia. Ecology and Society, 16(1), 30.

Biggs, D., Hall, C. M., \& Stoeckl, N. 2012. The resilience of formal and informal tourism enterprises to disasters: reef tourism in Phuket, Thailand. Journal of Sustainable Tourism, 20(5), 645-665.

Biggs, R., Schlüter, M., \& Schoon, M. L. (Eds.). 2015. Principles for building resilience: sustaining ecosystem services in social-ecological systems. Cambridge University Press.

Boarnet, M. G. 1996. Business losses, transportation damage and the Northridge earthquake. University of California Transportation Center.

Bowen, G. A. 2009. Document analysis as a qualitative research method. Qualitative research journal, $9(2), 27-40$

Briedenhann, J., \& Wickens, E. 2004. Tourism routes as a tool for the economic development of rural areas-vibrant hope or impossible dream?. Tourism management, 25(1), 71-79.

Brohman, J. 1996. New directions in tourism for third world development. Annals of tourism research, 23(1), 48-70.

Brown, E. D., \& Williams, B. K. 2015. Resilience and Resource Management. Environmental management, 56(6), 1416-1427.

Bruneau, M., Chang, S. E., Eguchi, R. T., Lee, G. C., O’Rourke, T. D., Reinhorn, A. M., \& von Winterfeldt, D. 2003. A framework to quantitatively assess and enhance the seismic resilience of communities. Earthquake spectra, 19(4), 733-752.

Bruner, E. M. 2005. Culture on tour: Ethnographies of travel. University of Chicago Press.

Cai, H., Lam, N. S. N., Zou, L., Qiang, Y., \& Li, K. 2016. Assessing Community Resilience to Coastal Hazards in the Lower Mississippi River Basin. Water, 8(2), 46.

Carpenter, S., Walker, B., Anderies, J. M., \& Abel, N. 2001. From metaphor to measurement: resilience of what to what?. Ecosystems, 4(8), 765-781.

Coburn, A. W., Spence, R. J. S., \& Pomonis, A. 1992, July. Factors determining human casualty levels in earthquakes: mortality prediction in building collapse. In Proceedings of the 10th World Conference on Earthquake Engineering (pp. 5989-5994).

Creswell, J. W., \& Clark, V. L. P. 2007. Designing and conducting mixed methods research.

Cutter, S. L. (2016). The landscape of disaster resilience indicators in the USA. Natural hazards, 80(2), 741-758.

Cutter, S. L., Barnes, L., Berry, M., Burton, C., Evans, E., Tate, E., \& Webb, J. 2008. A place-based model for understanding community resilience to natural disasters. Global environmental change, 18(4), 598-606.

Dahles, H., \& Susilowati, T. P. 2015. Business resilience in times of growth and crisis. Annals of Tourism Research, 51, 34-50.

Davoudi, S., Shaw, K., Haider, L. J., Quinlan, A. E., Peterson, G. D., Wilkinson, C., ... \& Davoudi, S. 2012. Resilience: A Bridging Concept or a Dead End?"Reframing” Resilience: Challenges for Planning Theory and Practice Interacting Traps: Resilience Assessment of a Pasture Management System in Northern Afghanistan Urban Resilience: What Does it Mean in Planning Practice? Resilience as a Useful Concept for Climate Change Adaptation? The Politics of Resilience for Planning: A Cautionary Note: Edited by Simin Davoudi and Libby Porter. Planning Theory \& Practice, 13(2), 299-333. 
De Sausmarez, N. 2007. Crisis management, tourism and sustainability: The role of indicators. Journal of sustainable tourism, 15(6), 700-714.

Dictionary, O. E., \& Barber, K. 2006. Oxford University Press. New York.

Du Cros, H., \& McKercher, B. 2014. Cultural tourism. Routledge.

Eugenio-Martín, J., Martín Morales, N., \& Scarpa, R. 2004. Tourism and economic growth in Latin American countries: A panel data approach.

Field, A. 2013. Discovering statistics using IBM SPSS statistics. Sage.

Folke, C. 2006. Resilience: The emergence of a perspective for social-ecological systems analyses. Global environmental change, 16(3), 253-267.

Folke, C., Carpenter, S., Elmqvist, T., Gunderson, L., Holling, C. S., \& Walker, B. 2002. Resilience and sustainable development: building adaptive capacity in a world of transformations. AMBIO: A journal of the human environment, 31(5), 437-440.

Glaesser, D. 2006. Crisis management in the tourism industry. Routledge.

Herrera, G., \& Rodríguez, G. 2016. RESILIENCIA Y TURISMO: EL CASO DE LA CIUDAD DE BAÑOS DE AGUA SANTA-

Holladay, P. J., \& Powell, R. B. 2013. Resident perceptions of social-ecological resilience and the sustainability of community-based tourism development in the Commonwealth of Dominica. Journal of Sustainable Tourism, 21(8), 1188-1211.

Holling, C. S. 1973. Resilience and stability of ecological systems. Annual review of ecology and systematics, 1-23.

Jopp, R., DeLacy, T., \& Mair, J. 2010. Developing a framework for regional destination adaptation to climate change. Current Issues in Tourism, 13(6), 591-605.

Leigh, N. G., \& Blakely, E. J. 2013. Planning local economic development: Theory and practice. SAGE Publications, Incorporated.

Lew, A. A. 2014. Scale, change and resilience in community tourism planning. Tourism Geographies, 16(1), 14-22.

Lew, A. A., Ng, P. T., Ni, C. C., \& Wu, T. C. 2016. Community sustainability and resilience: similarities, differences and indicators. Tourism Geographies, 18(1), 18-27.

Ludin, S. M., \& Arbon, P. A. 2017. Improving community disaster resilience through scorecard self-testing. Disaster Prevention and Management: An International Journal, 26(1), 13-27.

Magis, K. 2010. Community resilience: an indicator of social sustainability. Society and Natural Resources, 23(5), 401-416.

Mayunga, J. S. 2007. Understanding and applying the concept of community disaster resilience: a capital-based approach. Summer academy for social vulnerability and resilience building, 1, 16.

McKercher, B. 1993. The unrecognized threat to tourism: Can tourism survive 'sustainability?. Tourism management, 14(2), 131-136.

Neto, F. 2003, August. A new approach to sustainable tourism development: Moving beyond environmental protection. In Natural resources forum (Vol. 27, No. 3, pp. 212-222). Oxford, UK: Blackwell Publishing Ltd.

Norris, F. H., Stevens, S. P., Pfefferbaum, B., Wyche, K. F., \& Pfefferbaum, R. L. 2008. Community resilience as a metaphor, theory, set of capacities, and strategy for disaster readiness. American journal of community psychology, 41(1-2), 127-150.

Padgett, D. K. 2016. Qualitative methods in social work research (Vol. 36). Sage Publications.

Pallant, J. 2013. SPSS survival manual. McGraw-Hill Education (UK).

Paton, D., Millar, M., \& Johnston, D. 2001. Community resilience to volcanic hazard consequences. Natural hazards, 24(2), 157-169.

Pennington-Gray, L., Thapa, B., Kaplanidou, K., Cahyanto, I., \& McLaughlin, E. 2011. Crisis planning and preparedness in the United States tourism industry. Cornell Hospitality Quarterly, 52(3), 312-320.

Ritchie, B. W. 2004. Chaos, crises and disasters: a strategic approach to crisis management in the tourism industry. Tourism management, 25(6), 669-683.

Ruiz-Ballesteros, E. 2011. Social-ecological resilience and community-based tourism: an approach from Agua Blanca, Ecuador. Tourism Management, 32(3), 655-666.

Santana, G. 2004. Crisis management and tourism: Beyond the rhetoric. Journal of Travel \& Tourism Marketing, 15(4), 299-321.

Scheyvens, R. 2007. Exploring the tourism-poverty nexus. Current issues in tourism, 10(2-3), 231-254.

Schmidtlein, M. C., Shafer, J. M., Berry, M., \& Cutter, S. L. 2011. Modeled earthquake losses and social vulnerability in Charleston, South Carolina. Applied Geography, 31(1), 269-281. 
Sherrieb, K., Norris, F. H., \& Galea, S. 2010. Measuring capacities for community resilience. Social indicators research, 99(2), 227-247.

Sherrieb, K., Norris, F. H., \& Galea, S. 2010. Measuring capacities for community resilience. Social indicators research, 99(2), 227-247.

Strickland-Munro, J. K., Allison, H. E., \& Moore, S. A. 2010. Using resilience concepts to investigate the impacts of protected area tourism on communities. Annals of Tourism Research, 37(2), 499-519.

Strickland-Munro, J. K., Allison, H. E., \& Moore, S. A. 2010. Using resilience concepts to investigate the impacts of protected area tourism on communities. Annals of Tourism Research, 37(2), 499-519.

Sydnor-Bousso, S. B. 2009. Assessing the impact of industry resilience as a function of community resilience: The case of natural disasters (Doctoral dissertation, The Ohio State University).

Tobin, G. A. 1999. Sustainability and community resilience: the holy grail of hazards planning?. Global Environmental Change Part B: Environmental Hazards, 1(1), 13-25.

Walker, B. H., Carpenter, S. R., Rockstrom, J., Crépin, A. S., \& Peterson, G. D. 2012. Drivers," slow" variables," fast" variables, shocks, and resilience. Ecology and Society, 17(3), 30.

Walker, B., \& Salt, D. 2012. Resilience practice: building capacity to absorb disturbance and maintain function. Island Press.

Walker, B., \& Salt, D. 2012. Resilience thinking: sustaining ecosystems and people in a changing world. Island Press.

Walker, B., Holling, C. S., Carpenter, S. R., \& Kinzig, A. 2004. Resilience, adaptability and transformability in social--ecological systems. Ecology and society, 9(2), 5.

Walsh, F. 2007. Traumatic loss and major disasters: Strengthening family and community resilience. Family process, 46(2), 207-227. 